\title{
Models of COVID-19 vaccine prioritisation: a systematic literature search and narrative review
}

\author{
Nuru Saadi ${ }^{1^{*}}$ (D, Y-Ling Chi ${ }^{2}$, Srobana Ghosh ${ }^{2}$, Rosalind M. Eggo ${ }^{3}$, Ciara V. McCarthy ${ }^{3}$, Matthew Quaife ${ }^{3}$,
} Jeanette Dawa ${ }^{4,5}$, Mark Jit $^{3+}$ and Anna Vassall ${ }^{1 \dagger}$

\begin{abstract}
Background: How best to prioritise COVID-19 vaccination within and between countries has been a public health and an ethical challenge for decision-makers globally. We reviewed epidemiological and economic modelling evidence on population priority groups to minimise COVID-19 mortality, transmission, and morbidity outcomes.

Methods: We searched the National Institute of Health iSearch COVID-19 Portfolio (a database of peer-reviewed and pre-print articles), Econlit, the Centre for Economic Policy Research, and the National Bureau of Economic Research for mathematical modelling studies evaluating the impact of prioritising COVID-19 vaccination to population target groups. The first search was conducted on March 3, 2021, and an updated search on the LMIC literature was conducted from March 3, 2021, to September 24, 2021. We narratively synthesised the main study conclusions on prioritisation and the conditions under which the conclusions changed.

Results: The initial search identified 1820 studies and 36 studies met the inclusion criteria. The updated search on LMIC literature identified 7 more studies. 43 studies in total were narratively synthesised. 74\% of studies described outcomes in high-income countries (single and multi-country). We found that for countries seeking to minimise deaths, prioritising vaccination of senior adults was the optimal strategy and for countries seeking to minimise cases the young were prioritised. There were several exceptions to the main conclusion, notably that reductions in deaths could be increased if groups at high risk of both transmission and death could be further identified. Findings were also sensitive to the level of vaccine coverage.

Conclusion: The evidence supports WHO SAGE recommendations on COVID-19 vaccine prioritisation. There is, however, an evidence gap on optimal prioritisation for low- and middle-income countries, studies that included an economic evaluation, and studies that explore prioritisation strategies if the aim is to reduce overall health burden including morbidity.
\end{abstract}

Keywords: COVID-19, Vaccination, Mathematical modelling

\footnotetext{
* Correspondence: Nuru.Saadi@lshtm.ac.uk

${ }^{\dagger}$ Mark Jit and Anna Vassall are joint senior authors.

'Department of Global Health and Development, London School of Hygiene and Tropical Medicine, London, UK

Full list of author information is available at the end of the article
}

(c) The Author(s). 2021 Open Access This article is licensed under a Creative Commons Attribution 4.0 International License, which permits use, sharing, adaptation, distribution and reproduction in any medium or format, as long as you give appropriate credit to the original author(s) and the source, provide a link to the Creative Commons licence, and indicate if changes were made. The images or other third party material in this article are included in the article's Creative Commons licence, unless indicated otherwise in a credit line to the material. If material is not included in the article's Creative Commons licence and your intended use is not permitted by statutory regulation or exceeds the permitted use, you will need to obtain permission directly from the copyright holder. To view a copy of this licence, visit http://creativecommons.org/licenses/by/4.0/ The Creative Commons Public Domain Dedication waiver (http://creativecommons.org/publicdomain/zero/1.0/) applies to the data made available in this article, unless otherwise stated in a credit line to the data. 


\section{Background}

As of September 2021, over 6 billion vaccine doses have been administered, but vaccines are still in limited supply in many countries [1]. The question of which groups should be prioritised for vaccination within countries and between them has continued to present both a public health and an ethical challenge to decision makers [2].

The World Health Organization (WHO) Strategic Advisory Group of Experts on Immunisation (SAGE) working group on COVID-19 vaccines has provided guidance to countries on the prioritisation of groups for vaccination while supply is limited. The guidance, based on the WHO SAGE values framework for the allocation and prioritisation of COVID-19 vaccines, seeks to ensure equitable protection of human health across the globe, and in particular, among those experiencing the greatest risk and burden of COVID-19 [2, 3].
Epidemiological and economic models can provide an assessment of the potential health and broader societal impact of different prioritisation policies, and identify the optimal groups to prioritise for vaccination, given different public health objectives and scenarios. These results can be considered alongside other decision criteria to allocate vaccines both globally and within countries faced with a limited supply.

There was only a limited set of modelling results available to inform SAGE decision making at the end of 2020 (Fig. 1), but in early 2021, the evidence base greatly expanded. The model results available at that time were largely limited to high-income and high-transmission settings such as the United States of America (USA) and United Kingdom (UK). Models specifically addressing low- and middle-income countries, as well as lowtransmission settings, were not available.

Stage I (very limited vaccine availability, for $1-10 \%$ nat. pop.)

1a: Health workers at high to very high risk of acquiring and transmitting infection. 1b: Older adults

Stage II (limited vaccine availability, for $11-20 \%$ nat. pop.)

Older adults not covered in Stage I.

Groups with comorbidities

Sociodemographic groups at significantly higher risk of severe disease or death (depending on country context)

Health workers engaged in immunization delivery (routine and COVID-19).

High-priority teachers and school staff

Stage III (moderate vaccine availability, for $21-50 \%$ nat. pop.)

Remaining teachers and school staff.

Other essential workers outside health and education sectors

Pregnant women

Health workers at low to moderate risk of acquiring and transmitting infection

Personnel needed for vaccine production and other high-risk laboratory staff.

Social/employment groups at elevated risk of acquiring and transmitting infection because they are unable to effectively physically distance (depending on country context)

Fig. 1 WHO SAGE vaccine prioritisation recommendations under different supply scenarios during community transmission (adapted from the WHO sage roadmap for prioritising uses of COVID-19 vaccines in the context of limited supply) 
We aimed to systematically review the epidemiological and economic modelling literature on population groups to prioritise for COVID-19 vaccination to minimise COVID-19 mortality, transmission, and morbidity outcomes, in order to inform prioritisation policy at both the global and national levels. Our study is structured around the policy questions that SAGE considered in 2020. In summary, these questions examined exploring optimal allocation by age groups, occupational groups, groups with comorbidities, and groups at higher risk of infection, considering the impact on deaths, cases, morbidity, and economic outcomes [3].

\section{Methods}

\section{Search strategy and selection criteria}

The systematic literature review was performed in line with PRISMA guidelines (Fig. 2) [4]. We searched the
National Institute of Health (NIH) iSearch COVID-19 Portfolio on the 3rd of March 2021 (a database which sources peer-reviewed articles from PubMed and preprints from arXiv, bioRxiv, ChemRxiv, medRxiv, Preprints.org, Qeios, Research Square, and SSRN). We searched Econlit on the 3rd of March 2021, using the advanced filters to include studies published between 2020 and 2021. To search these databases, we used a Boolean strategy to combine keywords such as 'model"', 'vacc"', 'econom"', 'cost', and 'COVID-19'. We contacted the Centre for Economic Policy Research in the UK and the National Bureau of Economic Research in the USA and received their full datasets of studies on the economics of COVID-19. Because of the lack of low- and middle-income country (LMIC) studies in the first search, an updated search narrowed to LMIC literature was run on the 24th of September 2021. We also

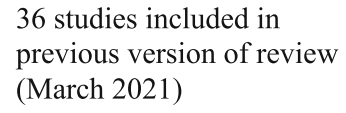

647 records identified through new search (September 2021)
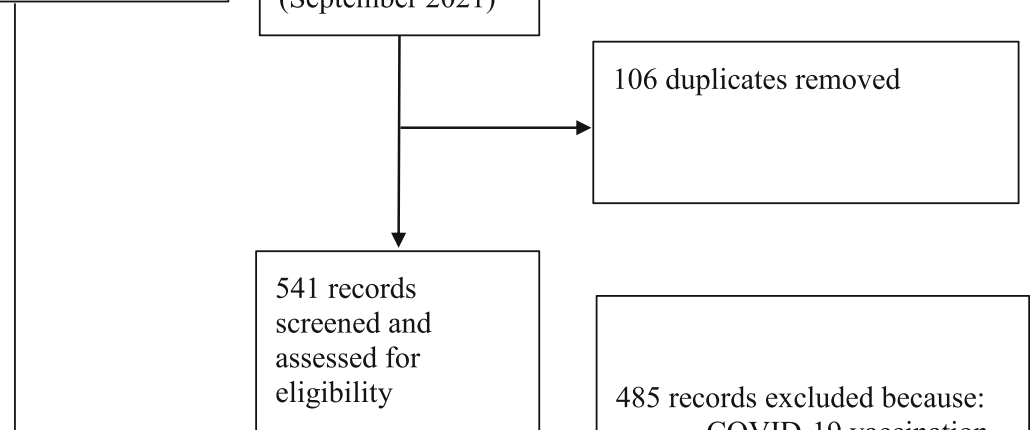

541 records assessed for eligibility

485 records excluded because:

- COVID-19 vaccination not explicitly modelled

- Modelled vaccination outcomes in the clinical setting

- Model purely theoretical

56 full-text articles assessed for eligibility

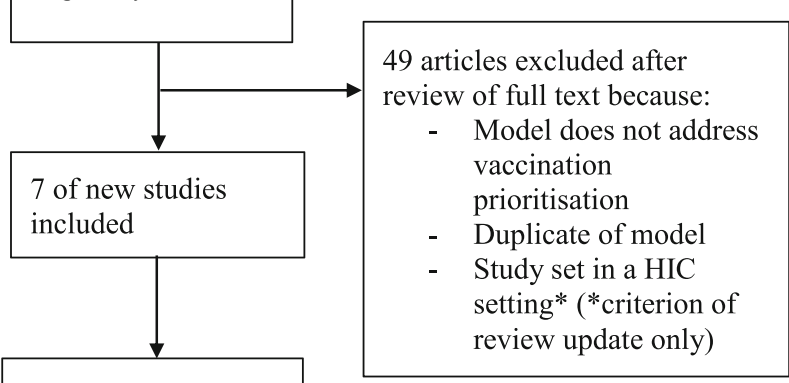

43 of studies included (total) 
narrowed the search terms for the updated search to retrieve more relevant studies based on the experience of the first search (Additional file 1: Table S1 for the full search strategy and further details).

We included English language published or pre-print studies that used mathematical modelling to assess the impact of prioritising population target groups (either within or between countries) for COVID-19 vaccination on mortality, health (e.g. cases, quality adjusted life years), health care (e.g. hospitalisations), and/or economic (e.g. costs and cost-effectiveness) outcomes. We excluded studies which did not use a mathematical model to project the impact of COVID-19 vaccination, where none of the parameters were determined by empirical data (e.g. theoretical, non-empirical models), or which modelled vaccination outcomes within a clinical trial or a within-country small local setting, such as a care home, rather than nation or district wide allocation. For the updated search for LMIC literature, we applied an additional criterion of excluding HIC studies.

Two researchers independently screened titles and abstracts during the first round of screening. During the second round, three researchers independently screened titles/abstracts and full-text articles. All studies were screened by at least two reviewers, where there were disagreements about inclusion these were resolved in discussion with a fourth researcher. Three groups of two researchers each independently extracted the data from the included studies, with at least two groups reviewing each study. Discordant entries between the sets of extraction sheets were resolved by discussion between the groups. Data were recorded in Microsoft Excel files summarising the policy objectives, outcomes, characteristics of the studies, the study conclusions and the conditions under which the conclusions changed, i.e., sensitivity analyses (see Additional file 2: full extraction sheet).

Studies explored the optimal prioritisation strategy based on different policy objectives/outcomes (deaths, cases etc.). We therefore extracted data and organised our results tables by the objective used. Some studies used two objectives to inform prioritisation (for example cases and deaths). In these instances, we extracted and synthesised both sets of results. A full list of outcomes modelled in the included studies can be found in Table 1. We referred to the Economic and Social Research Council's guidance on the conduct of narrative synthesis to aid data synthesis [5]. We therefore organised and grouped the textual results of the studies so that we could identify patterns within and between them. Synthesis was organised by the outcomes being explored. Due to wide variation between the studies in age group boundaries and other group classifications, extracted data from the study conclusions on vaccination priority groups were re-classified into broader population categories to aid synthesis. The population group categories considered were children/adolescents (ages 018 ), young adults (ages 19-40), middle-aged adults (ages 41-64), seniors (65+), groups with comorbidities, high social contact groups, essential workers, health workers, and geographic regions.

Studies had different combinations of comparators, so we present results specifying the full range of comparator populations assessed. Study setting was categorised as high-income (HIC), upper middle-income (UMIC), lower-middle income (LMIC), low income (LIC), multiregion, or not specified, using World Bank classifications for 2021 [6]. We extracted the modelling methods used, and reviewed assumptions and model structure in detail for the studies that did not align with the majority of conclusions to identify if this was based on an exceptional method (referred to as 'exceptions'). We also report where sensitivity analyses generated results that were contrary to main findings of the study.

\section{Results}

In the first search, our database search returned 2279 studies. After the removal of 459 duplicates, 1820 records were included in the title and abstract screening. After title and abstract screening, 55 studies remained for full-text screening. After assessing the full text of the 55 studies for eligibility, 36 studies were included in the systematic review. After updating the search to look for studies set in LMIC countries having identified this as the predominant gap in the literature, 7 more studies were included (Fig. 2).

Study characteristics are summarised in Table 1. All the reviewed studies used models that captured transmission between individuals, with deterministic compartmental models being most common (28/43). However, agent-based models (6/43), stochastic compartmental models $(4 / 43)$, a delay differential equation model (1/43), and a linear model (1/43) were also used. Studies most commonly used a SEIR (Susceptible, Exposed, Infected, Recovered) (12/43) or Expanded SEIR (19/43) natural history. Most of them were set in a HIC (26/43); there were few singlecountry UMIC (3/43) and LMIC (5/43) studies. There were no single-country studies in a LIC setting. Only a few (6/43) looked at more than one country and two did not explicitly state the study setting. Most studies explored multiple policy objectives/outcomes regarding prioritisation: $34 / 43$ investigated strategies to minimise deaths, $27 / 43$ investigated minimisation of cases, 11/43 hospitalisations, 1/43 quality adjusted life years (QALYs), 1/43 disability adjusted life years (DALYs), and 3/43 years of life lost (YLLs). Only 2/ 43 considered economic outcomes, such as financial or economic costs, in relation to prioritisation. 
Table 1 Characteristics of all studies included in the narrative synthesis

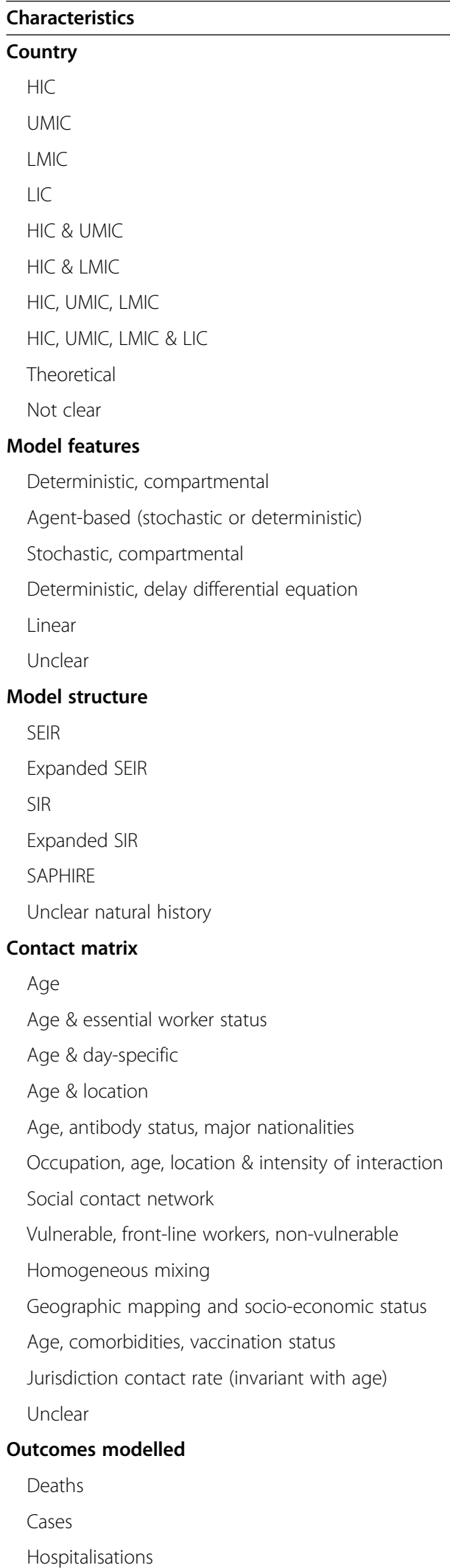

Table 1 Characteristics of all studies included in the narrative synthesis (Continued)

\begin{tabular}{ll}
\hline Characteristics & (n) \\
\hline ICU admission & 6 \\
No of vx to avert one infection & 1 \\
Loss of economic benefits & 1 \\
Years of life lost & 3 \\
QALYs & 2 \\
DALYs & 1 \\
Cost-effectiveness ratio & 1 \\
Net present value of damages (VSL \& DALYs) & 1 \\
Infection attack rate & 2 \\
Peak infections & 1 \\
Risk of new wave & 1 \\
Life-years gained & 1 \\
Total cost & 2 \\
Net monetary benefits & 1 \\
Effective reproductive number & 1 \\
Herd immunity & 1 \\
\hline
\end{tabular}

\section{Prioritisation to minimise deaths}

Table S2 (Additional file 1) summarises the study conclusions highlighting the priority group and all the comparators included in each study (see the 'Methods' section for how we defined population group categories). Most studies included seniors in the priority group. Nineteen studies recommended that seniors should be prioritised for vaccination to minimise deaths [7-25]. One study recommended prioritising seniors with comorbidities [26], and six studies recommended vaccinating seniors at the same time as another priority group (middle-aged adults, the highest social contact group, young and middle-aged adults who are in high contact with them, young adults with partial vaccine dose, and health workers) [27-32].

Ten studies did not find that prioritising the groups at highest risk of mortality (seniors or people living with comorbidities) minimised deaths (for a summary of these studies see Additional file 1: Table S3) [29, 30, 3340]. These 'exceptional' studies instead found that prioritising groups with a higher risk of infection would lead to fewer deaths; in other words, that the indirect protection from lower transmission outweighs the benefits from direct protection from vaccines for those at the highest risk of mortality. The group at high risk of infection were defined as those with higher contact rates, e.g. a synthetic population with 3-10 times the average number of contacts of the age groups 30-39 [29], individuals with an expansive social network [37, 38], and individuals with essential worker status [33, 34]. In addition, two studies defined young adults as the group 
with the highest social interactions and therefore at higher risk of infection [35, 40]. One study examined vaccination of individuals that had high levels of interaction with seniors [30].

One of the ten exceptional studies concluded that the non-vulnerable group should be prioritised for vaccination compared to the group with comorbidities [36]. In this study, the authors state they assumed that 'the time required to vaccinate the vulnerable group is identical to that of the much larger non-vulnerable group'. Finally, one study recommended prioritising vaccination through the use of serological testing to achieve the greatest reduction in COVID-19-related deaths [41].

\section{Prioritisation to minimise COVID-19 cases}

Table S4 (Additional file 1) summarises the study conclusions. The largest proportion of the selected studies $(N=27)$ investigated optimal vaccine prioritisation strategies to minimise COVID-19 cases. Of these, seven studies recommended young and middle-aged adults [10, 12, $15,16,17,20,28]$, three young adults [23, 25, 39], and two young adults and children [27, 32]. One study recommended young people at the same time as another priority group (seniors at full vaccine dose and young adults at partial dose) [31].

Seven studies recommended vaccination prioritisation based on social or occupational interactions compared to age group prioritisation [14, 33, 34, 37, 38, 42, 43]. Of these, three studies recommended prioritising essential workers to minimise cases [14, 33, 34], and four studies recommended prioritising high social contact adults compared to other age groups [37, 38, 42, 43].

Two studies recommended prioritising vaccination using serological testing to prioritise antibodynegative individuals compared to not using serological testing [41, 44]. Two studies investigated allocation between geographic areas of disease burden-the first recommended that the geographic area with lower disease burden should be prioritised for vaccination, whereas the second recommended that the geographic area with the highest disease burden should be prioritised [45-46].

There were a few studies concluding differently to the majority recommendations on minimising cases (for a summary of these studies see Additional file 1: Table S5). Three studies found that scenarios targeting seniors $[13,18,31]$ led to the highest reduction in cases. However, two of those studies did not have a comparator that modelled those strategies comparted to more socially interactive populations [18, 31]. Chhetri et al. found very small differences between scenarios, and the conclusion was not reported in the "Results" section [13].

\section{Prioritising other outcomes}

Studies investigating strategies to minimise hospitalisations from COVID-19 tended to reach similar conclusions to studies investigating deaths $(N=11)$. Eight studies recommended prioritising seniors $[8,15,25,16,43,47]$, senior- and middle-aged adults [27], or seniors and the high social contact group [29] for vaccination compared to other age and occupational groups. Four studies concluded differently from the majority of the hospitalisation outcome studies [37, 38, 44, 48]. Two recommended prioritising the high social contact group compared to prioritising senior adults [37, 38]. One study recommended prioritising vaccination by serological testing compared to no serological testing [44]. One study recommended giving equal priority to all age and risk groups compared to a targeted age-based prioritisation [48].

A few studies investigated the optimal vaccination strategy when maximising QALYs, DALYs, or YLLs. One study modelled a vaccination prioritisation strategy to minimise QALY losses [11]. The authors concluded that the most effective strategy to minimise QALY losses is to prioritise seniors for vaccination compared to other age groups, groups with comorbidities, and no group prioritisation. Three studies investigated within-country vaccine prioritisation strategies for minimising YLLs [12, 28, 34]. Two studies recommended prioritising seniors for vaccination to minimise YLLs [12, 34], and the other recommended prioritising middle aged adults and seniors [28]. One study modelled the impact of COVID-19 vaccination on DALYs [19]. The authors found that the amount of DALYs averted under a base vaccination strategy which prioritised seniors was stable to a scenario where everyone over 15 years old is vaccinated [19].

One study considered the cost-effectiveness of COVID-19 vaccination [19]. The authors found that the strategy of prioritising seniors for vaccination was similarly cost-effective to vaccinating all individuals over 15 years old [19].

One study investigated prioritisation strategies for optimising the incremental net monetary benefit (iNMB) of vaccination, i.e. the net economic gain from vaccination including both costs saved and monetised health gains [48]. The authors concluded that giving equal priority to all age and risk groups was most optimal compared to prioritising seniors, high risk individuals, and both seniors and high-risk individuals when vaccine effectiveness was only moderate $(40 \%)$ and coverage was low (20\%). Conversely, when vaccine effectiveness was high $(80 \%)$ and coverage was moderate $(50 \%)$, vaccinating high risk individuals resulted in the highest iNMB.

\section{Prioritisation by setting}

Five of the included articles were single-country studies modelling LMIC settings $[14,17,19,22,46]$. Four 
studies modelled UMIC settings [21, 23, 25, 33]. These studies reached the same conclusions as the HIC studies i.e. studies minimising deaths recommended prioritising seniors, while those minimising cases recommended prioritising high transmission groups. The exception was one study from Thailand on minimising cases which recommended prioritising high transmission groups to minimise deaths [39].

There were also five multi-country studies which modelled LMIC settings [8, 9, 12, 20, 24] and two modelling UMIC settings [20,29]. The conclusions for these studies were in line with the majority conclusions for deaths and cases (except for one study which recommended prioritising both the high social contact group and seniors to minimise deaths) [29]. See Additional file 1: Table S6 for a summary of the studies modelling UMIC and LMIC settings.

One study also considered prioritisation between countries, in addition to within countries. This study made recommendations on global vaccine allocation strategies to optimise different health objectives [9]. The authors concluded that the optimal strategy to minimise deaths was to allocate doses equitably across all income settings relative to population size and then to prioritise vaccination of seniors within countries. This performed better than allocating vaccines to countries based on their respective senior population sizes, giving preferential allocation to HICs, giving preferential allocation to LICs and LMICs, or allocating doses in proportion to population plus providing a set number of extra doses to HIC and UMICs [9]. When YLLs were used as an optimisation outcome measure, LMIC settings received the most doses.

\section{Factors that influence prioritisation strategy}

40 out of 43 (93\%) studies included a sensitivity analysis (see Additional file 1: Table S7 for a summary of these studies). Of these, 17 studies reported a sensitivity analysis that led to a potential change in the recommended prioritisation strategy. While there were a wide range of parameters tested in the uncertainty analysis, there were only a few that consistently drove a change in prioritisation. The most common parameters that influenced prioritisation all related to vaccine coverage, i.e. level of vaccine supply, coverage, and speed of rollout (see Additional file 1: Table S8 for a summary of coverage level assumptions made by the exceptional studies to the majority of the study conclusions). Transmission rates and vaccine efficacy were also considered.

Eight studies reported that the trade-off between direct and indirect protection is sensitive to the proportion of people vaccinated $[9,12,15,22,27,28,38,25]$. These papers stated that when vaccine supply is very low, vaccination has a minimal impact on interrupting transmission, so more deaths can be prevented by vaccinating groups at risk of severe disease (e.g. essential workers, seniors, and clinical risk groups). However, as supply increases, this opens up the possibility of interrupting transmission, which shifts the optimal policy for preventing deaths to prioritising the young or those with many contacts. If there is very high vaccine supply, seniors are again favoured for prioritisation if aiming to reduce deaths, as there is sufficient coverage to achieve both direct protection of the most vulnerable and indirect protection of key transmitters. One study stated that direct effects of immunisation take precedence in deciding prioritisation when the vaccine supply is sufficient to cover the priority groups in the study which make up $18 \%$ of the population (key workers, individuals with comorbidities, and the over-60s) [22].

The influence of COVID-19 transmission rates was reported often in LMICs, but with varied results. One study in India suggested that when the transmission rate is low, those with comorbidities should be prioritised over those aged over 60 years old [22]. In Brazil, modelling results suggested that the impact on deaths of vaccinating the young increases with earlier vaccination dates, lower vaccine efficacy, and higher transmission rates [23]. In Columbia, the presence of the Delta variant reduced the magnitude of difference in the impact of vaccinating different groups. However, in all papers, the base-case result was to prioritise seniors [21].

Several studies tested different values of vaccine efficacy, with most reporting before full results of vaccine trials became available starting in late 2020. Generally, variations in vaccine efficacy did not appear to change prioritisation unless efficacy was significantly lower in older rather than younger populations. However, a number of studies assumed that vaccines had similar levels of efficacy against severe disease, infection, or transmission. Where vaccines were more efficacious against severe disease strategies, the priority was to vaccinate highest transmitters.

\section{Discussion}

We find that for countries seeking to minimise deaths, the current evidence base supports prioritising vaccination of seniors $(65+)$ as the optimal strategy, unless there are exceptional cases where specific non-agerelated high-risk groups or very highly networked individuals can be identified and prioritised. The difference in deaths averted can be large between depending on the strategies, for example in this study, a symptomblocking vaccine with $50 \%$ uptake prioritising seniors and high-risk groups averted 17,000 more deaths than an unprioritised campaign [26]. For countries seeking to minimise cases, the evidence supports prioritising young age groups and essential workers. The evidence base 
examining the optimal strategy to improve health in general is too limited to draw any firm conclusions. See Additional file 1: Table S9 for a table showing how much of the evidence base supported the WHO SAGE vaccine prioritisation roadmap.

While in principle prioritising highly socially connected groups may be optimal to reduce mortality, it could prove difficult in practice to identify these groups, especially when their definition and means of identification are only vaguely defined $[29,30,37,38]$. Chen et al. suggest those population groups could be identified through contact tracing, while recognising the limitations of such an approach in resource constrained settings [37]. Santini recommends prioritising younger people with many connections to vulnerable people [30]. Buckner et al. find that prioritising essential workers (based on occupation) could lead to fewer deaths in the context of strong non-pharmaceutical interventions [34]. As no studies included the feasibility and costs of identifying and delivering vaccines to highly connected groups, it is unclear whether prioritisation to groups that are not age or occupation-based is possible or cost-effective.

A study in Thailand examined how vaccines may be prioritised in a low-incidence setting [39], finding that prioritising younger age groups would lead to greater reductions in deaths. This is similar to the study in India which found that when transmission was low, the optimal group to prioritise to reduce deaths shifted from older people to those with comorbidities to increase indirect effects on transmission [22]. With two studies modelling a low-incidence scenario in our sample, more research may be required to validate this finding across settings and modelling approaches. The small number of studies set in low- and middle-income countries, and the lack of evidence on the cost-effectiveness of reaching different populations also limits our findings. Although we found that most of the studies modelling these settings were in line with the conclusions from studies set in HIC for minimising deaths and cases, context may impact results, especially when very limited supply is considered. The studies based in LMIC settings assumed a higher level of vaccine supply available and level of coverage achieved than has been observed in most LMICs (Additional file 1: Table S6). Further research is urgently required to model the effect of different levels of supply on prioritisation in lower income settings.

There was only one study modelling inter-country allocation of vaccines [9], despite the political importance of this issue [49]. That one study found that doses should be allocated equally by the population size if minimising deaths and allocated preferentially to lowand middle-income countries if optimising life-years gained. Since this differs drastically from the current allocation of vaccine doses globally, it points to the need for further investigation and action [9].

There were two studies modelling allocation of vaccines between geographical areas within the same country $[45,46]$. One study concluded that geographical areas of high disease burden should be prioritised for vaccination [46], while the other concluded that the geographical areas of low disease burden should be prioritised [45]. The author's interpretation of the latter result is that in a scenario where vaccine supplies were low and there was non-compliance to travel restrictions between geographical areas, the area of lower disease burden was prioritised as it had a greater proportion of susceptible individuals.

Only one study incorporated the impact of variants of concern on vaccination prioritisation [21], possibly because most of our review focused on the time period up to March 2021 only. The authors of this study in Colombia were modelling the impact of age-based prioritisation strategies of vaccination (oldest first) in the context of variants and social mixing. In a scenario where the delta variant became dominant after midSeptember 2021, age prioritisation of vaccination became less important than if delta became dominant by mid-July due to higher levels of vaccine coverage. However, maintaining a 21-day interval between the first and second dose without further delay became more important in the September scenario due to the characteristics of the delta variant [21].

Our review found that the optimal prioritisation strategy to pursue depends on the public health objective(s) of the decision-maker, with different conclusions depending on whether the objective is reducing cases or reducing mortality $[37,38]$. The trade-offs between different objectives are a challenging ethical issue for decision-makers.

The WHO SAGE value framework for the allocation and prioritisation of vaccines proposed 6 principles as the ethical basis of decisions on vaccine prioritisation: the promotion of human well-being, equal respect, global equity, national equity, reciprocity, and legitimacy [2]. Within the framework, reducing disease burden overall (and not purely the number of deaths) is a key consideration to promote human wellbeing. However, only one study considered integrated burden of disease outcomes such as QALYs that combine both morbidity and mortality in relation to prioritisation [11], one study considered DALYs [19]. This evidence gap may be particularly limiting in settings with a younger population, such as in many low- and middle-income countries, where overall mortality may be a smaller proportion of the overall COVID-19-related burden morbidity compared to high-income countries. 
Only one study considered within-country equity (such as prioritising populations that have suffered disproportionately from COVID-19 because of their socioeconomic status) [43]. We also consider the few economic studies, such as economic evaluations, to represent a research gap. The choice of one vaccine strategy over another in the studies evaluated often only took into account the net health gain, yet the choice of the most appropriate vaccination strategy should take into account health benefits, costs, and the willingness to pay threshold-which varies in each setting [50].

Our findings are limited by several methodological issues. By limiting our search to English language studies, we may have missed relevant studies, particularly in low- and middle-income countries. Much of this literature is pre-print studies which are not peerreviewed, so the quality of the evidence presented here should be viewed with caution. Furthermore, the included studies mainly reflect the earlier stage of the pandemic up to March 2021, and variants of concern were not well accounted for in the modelling literature at that time. This may have had implications on which group to prioritise for vaccination considering differences of transmissibility between variants. Finally, to highlight key findings across all studies, we categorised the reviewed studies according to the broad public health objectives that they aimed to fulfil. However, this categorisation may have obscured some nuances within studies, such as where there were variations in study conclusions grouped under the same category.

\section{Conclusion}

The findings of this systematic literature review have provided empirical evidence for the prioritisation of seniors for vaccination to minimise COVID-19 deaths and young people to minimise COVID-19 transmission. However, there remain critical gaps in the evidence around strategies that reduce overall health outcomes, considering the costs of different prioritisation strategies and for low- and middle-income settings. The research gaps identified can help to guide the direction of further research on vaccination prioritisation as the pandemic continues to evolve.

\footnotetext{
Abbreviations

COVID-19: Coronavirus diseases 2019; QALY: Quality-adjusted life-year; DALY: Disability-adjusted life-year; WHO SAGE: World Health Organization Strategic Advisory Group of Experts; LMIC: Lower middle income country; UMIC: Upper middle income country; HIC: High-income country; YLL: Years of life lost; iNMB: Incremental Net Monetary Benefit
}

\section{Supplementary Information}

The online version contains supplementary material available at https://doi. org/10.1186/s12916-021-02190-3.
Additional file 1. Models of COVID-19 vaccine prioritisation: a systematic literature search and narrative review includes Tables S1 to S9. Table S1 - Search strategy. Table S2 - Conclusions of studies on optimal vaccination priority group to minimise deaths from COVID-19 with study comparators. Table $\mathbf{S 3}$ - Exceptions to the majority of study conclusions on optimal vaccination priority group to minimise deaths from COVID-19. Table S4 - Conclusions of studies on optimal vaccination priority group to minimise cases of COVID-19 with study comparators. Table S5 - Exceptions to the majority of study conclusions on optimal vaccination priority group to minimise cases from COVID-19. Table S6 - Characteristics of the included UMIC and LMIC studies including supply and coverage assumptions made. Table S7 - Summary of the results of the sensitivity analyses conducted by the included studies and whether this influenced the recommendation of prioritisation strategy. Table $\mathbf{S 8}$ - Exceptions to the majority of study conclusions on optimal vaccination priority group to minimise deaths and cases of COVID-19 organised by level of coverage by the end of the modelled vaccination campaign. Table S9 - Summary of evidence from the systematic literature review supporting the prioritisation of groups in the WHO SAGE vaccine prioritisation roadmap under different supply scenarios during community transmission.

Additional file 2. Full extraction sheet for the studies included in the narrative synthesis.

\section{Acknowledgements}

Not applicable

\section{Authors' contributions}

NS searched the literature. NS, YLC, SG, and AV reviewed the studies. NS, YLC, SG, RME, CM, MQ, JD, MJ, and AV did the data extraction. NS, YLC, SG, $\mathrm{MJ}$, and AV analysed the data. NS was the lead author, and NS, YLC, and SG wrote the first draft of the manuscript. All authors contributed to the data interpretation and to critical revision of the manuscript. The authors read and approved the final manuscript.

\section{Funding}

MJ was funded by the Bill \& Melinda Gates Foundation (grant: INV-003174, INV-016832), National Institute for Health Research (grant: 16/137/109, NIHR200929, NIHR200908), and European Union's SC1-PHE-CORONAVIRUS2020 programme (project EpiPose, grant: 101003688). YLC and SG were funded by the Bill \& Melinda Gates Foundation (grant: OPP1202541 iDSIPlus). AV and NS were funded by the Bill \& Melinda Gates Foundation (grant: OPP1202541 COVID IDSI). RME was funded by HDR UK (grant: MR/S003975/ 1), MRC (grant: MC_PC 19065), and NIHR (grant: NIHR200908).

Availability of data and materials All data generated or analysed during this study are included in this published article [and it's supplementary files].

\section{Declarations}

Ethics approval and consent to participate Not applicable.

\section{Consent for publication \\ Not applicable.}

\section{Competing interests}

The authors declare that they have no competing interests.

\section{Author details}

'Department of Global Health and Development, London School of Hygiene and Tropical Medicine, London, UK. ${ }^{2}$ International Decision Support Initiative, Center for Global Development, London, UK. ${ }^{3}$ Centre for Mathematical Modelling of Infectious Diseases, London School of Hygiene and Tropical Medicine, London, UK. ${ }^{4}$ Washington State University - Global Health Program, Nairobi, Kenya. ${ }^{5}$ Center for Epidemiological Modelling and Analysis, University of Nairobi, Nairobi, Kenya. 


\section{Received: 9 August 2021 Accepted: 17 November 2021} Published online: 01 December 2021

\section{References}

1. WHO Coronavirus (COVID-19) Dashboard. https://covid19.who.int/. Accessed 20 Sept 2021

2. World Health Organization. WHO SAGE values framework for the allocation and prioritization of COVID-19 vaccination. https://www.who.int/publica tions/i/item/whosage- values-framework-for-the-allocation-and-prioritizationof-covid-19-vaccination. Accessed 8 June 2021

3. World Health Organization. WHO Strategic Advisory Group of Experts (SAGE) on Immunization Working Group on COVID-19 Vaccines: Prioritized Infectious Disease and Economic Modelling Questions. https://www.who. int/immunization/policy/sage/SAGE_WG_COVID19_Vaccines_Modelling_ Questions 31July2020.pdf. Accessed 8 June 2021.

4. Liberati A, Altman DG, Tetzlaff J, Mulrow C, Gøtzsche PC, loannidis JPA, et al. The PRISMA statement for reporting systematic reviews and meta-analyses of studies that evaluate healthcare interventions: explanation and elaboration. BMJ. 2009;339:b2700. https://doi.org/10.1136/bmj.b2700.

5. Popay J, Roberts H, Sowden A, Pettricrew M, Arai L, Rodgers M et al. Guidance on the Conduct of Narrative Synthesis in Systematic Reviews. https://www.lancaster.ac.uk/media/lancaster-university/contentassets/ documents/fhm/dhr/chir/NSsynthesisguidanceVersion1-April2006.pdf. Accessed 8 June 2021.

6. World Bank Country and Lending Groups. https://datahelpdesk.worldbank. org/knowledgebase/articles/906519-world-bank-country and-lendinggroups. Accessed 8 June 2021.

7. Jentsch P, Anand M, Bauch CT. Prioritising COVID-19 vaccination in changing social and epidemiological landscapes. medRxiv. https://doi.org/1 0.1101/2020.09.25.20201889.

8. Meehan MT, Cocks DG, Caldwell JM, Trauer JM, Adekunle Al, Ragonnet RR, et al. Agetargeted dose allocation can halve COVID-19 vaccine requirements. medRxiv. https://doi.org/10.1101/2020.10.08.20208108.

9. Hogan BA, Winskill P, Watson OJ, Walker PGT, Whittaker C, Baguelin M et al. Report 33: Modelling the allocation and impact of a COVID-19 vaccine. https://www.imperial.ac.uk/mrc-global-infectious-disease-analysis/covid-19/ report-33-vaccine/. Accessed 8 June 2021.

10. Chen X, Li M, Simchi-Levi D, Zhao T. Allocation of COVID-19 Vaccines Under Limited Supply. medRxiv. https://doi.org/10.1101/2020.08.23.20179820.

11. Moore S, Hill EM, Dyson L, Tildesley MJ. Keeling MJ, Modelling optimal vaccination strategy for SARS-CoV-2 in the UK. medRxiv. https://doi.org/1 0.1101/2020.09.22.20194183.

12. Bubar KM, Kissler SM, Lipsitch M, Cobey S, Grad Y. Larremore DB, Modelinformed COVID-19 vaccine prioritization strategies by age and serostatus. medRxiv. https://doi.org/10.1101/2020.09.08.20190629.

13. Chhetri B, Vamsi DKK, Balasubramanian S. Sanjeevic CB, Optimal Vaccination and Treatment Strategies in Reduction of COVID-19 Burden. arXiv. https://a rxiv.org/abs/2102.09802v1.

14. Minoza JMA, Bongolan VP, Rayo JF. COVID-19 Agent-Based Model with Multiobjective Optimization for Vaccine Distribution. arXiv. https://arxiv.org/a bs/2101.11400v1.

15. Hoertel N, Blachier M, Limosin F, Sanchez-Rico M, Blanco C, Olfson M, et al. Optimizing SARS-CoV-2 vaccination strategies in France: Results from a stochastic agent-based model. medRxiv. https://doi.org/10.1101/2021.01.1 7.21249970 .

16. Tran TNA, Wikle N, Albert J, Inam H, Strong E, Brinda K, et al. Optimal SARSCoV-2 vaccine allocation using real-time seroprevalence estimates in Rhode Island and Massachusetts. medRxiv. https://doi.org/10.1101/2021.01.12.2124 9694.

17. Foy BH, Wahl B, Mehta K, Shet A, Menon Gl, Britto C. Comparing COVID-19 vaccine allocation strategies in India: a mathematical modelling study. medRxiv. https://doi.org/10.1101/2020.11.22.20236091.

18. Bertsimas D, Ivanhoe J, Jacquillat A, Li M, Previero A, Lami OS. et al, Optimizing Vaccine Allocation to Combat the COVID-19 Pandemic. medRxiv. 2020. https://doi.org/10.1101/2020.11.17.20233213.

19. Pearson $C A B$, Bozzani F, Procter SR, Davies NG, Huda M, Jensen HT, et al. Health impact and cost-effectiveness of COVID-19 vaccination in Sindh Province Pakistan. medRxiv. https://doi.org/10.1101/2021.02.24.21252338.

20. Gozzi N, Bajardi P, Perra N. The importance of non-pharmaceutical interventions during the COVID-19 vaccine rollout. PLoS Comput Biol. 2021; 17(9):e1009346. https://doi.org/10.1371/journal.pcbi.1009346.
21. España G, Cucunuba ZM, Cuervo-Rojas J, Diaz H, González-Mayorga M, Ramirez JD. The impact of vaccination strategies for COVID-19 in the context of emerging variants and increasing social mixing in Bogotá, Colombia: a mathematical modelling study. medRxix. https://doi.org/10.11 01/2021.08.06.21261734.

22. Mandel S, Arinaminpathy N, Bhargava B, Samiran P. India's pragmatic vaccination strategy against COVID-19: a mathematical modelling-based analysis. BMJ Open. 2021;11:e048874. https://doi.org/10.1136/bmjopen-2021048874

23. Campos EL, Cysne RP, Madureira AL, GLQ M. Multi-generational SIR modeling: Determination of parameters, epidemiological forecasting and age-dependent vaccination policies. Infect Dis Model. 2021;6:751-65. https://doi.org/10.1016/j.idm.2021.05.003.

24. Goldstein JR, Cassidy T, Mahmud AS. Lives Saved from Age-Prioritized Covid-19 Vaccination. medRxiv. https://doi.org/10.1101/2021.03.19.21253991.

25. Yu H, Han S, Cai J, Yang J, Zhang J, Wu Q, et al. Dynamic optimization of COVID-19 vaccine prioritization in the context of limited supply. Res Square. https://doi.org/10.21203/rs.3.rs-257573/v1.

26. Wang X, Du Z, Johnson KE, Pasco RF, Fox SJ, Lachmann M. et al, The impacts of COVID-19 vaccine timing, number of doses, and risk prioritization on mortality in the US. medRxiv. https://doi.org/10.1101/2021.01.18.212 50071.

27. Matrajt L, Eaton J, Leung T, Brown ER. Vaccine optimization for COVID-19, who to vaccinate first? medRxiv. https://doi.org/10.1101/2020.08.14.20175257.

28. Shim E. Optimal Allocation of the Limited COVID-19 Vaccine Supply in South Korea. J Clin Med. 2021;10(4):591.

29. Moret M, Filho TR, Mendes J, Murari T, Filho AN, Cordeiro A. et al, WHO vaccination protocol can be improved to save more lives. Res Square. https://doi.org/10.21203/rs.3.rs-148826/v1

30. Santini S. Covid-19 vaccination strategies with limited resources -- a model based on social network graphs. arXiv. https://arxiv.org/abs/2010.05312v1.

31. Hunziker P. Impact of personalized-dose vaccination in Covid-19 with a limited vaccine supply in a 100 day period in the U.S.A. medRxiv. https:// doi.org/10.1101/2021.01.30.21250834

32. Macintyre CR, Costantino V, Trent M. Modelling of COVID-19 vaccination strategies and herd immunity, in scenarios of limited and full vaccine supply in NSW, Australia. medRxiv. https://doi.org/10.1101/2020.12.15.20248278.

33. Babus A, Das S, Lee S. The Optimal Allocation of Covid-19 Vaccines. medRxiv. https://doi.org/10.1101/2020.07.22.20160143.

34. Buckner JH, Chowell G, Springborn MR. Dynamic Prioritization of COVID-19 Vaccines When Social Distancing is Limited for Essential Workers. medRxiv. https://doi.org/10.1101/2020.09.22.20199174.

35. Rodríguez J, Patón M, Acuña JM. COVID-19 vaccine prioritisation to the groups with the most interactions can substantially reduce total fatalities. medRxiv. https://www.medrxiv.org/content/10.1101/2020.10.12.20211094v2.

36. Bonsall MB, Huntingford $C$, Rawson T. Optimal time to return to normality: parallel use of COVID-19 vaccines and circuit breakers. medRxiv. https://doi. org/10.1101/2021.02.01.21250877

37. Chen J, Hoops S, Marathe A, et al. Prioritizing allocation of COVID-19 vaccines based on social contacts increases vaccination effectiveness. medRxiv. https://doi.org/10.1101/2021.02.04.21251012.

38. Goldenbogen B, Adler SO, Bodeit O, Wodke JAH, Escalera-Fanjul X, Korman A, et al. Optimality in COVID-19 vaccination strategies determined by heterogeneity in humanhuman interaction networks. medRxiv. https://doi. org/10.1101/2020.12.16.20248301.

39. Luangasanatip N, Pan-Ngum W, Prawjaeng J, Saralamba S, White L, Aguas R, et al. Optimal vaccine strategy to control COVID-19 pandemic in middleincome countries: Modelling case study of Thailand. Res Square. https://doi. org/10.21203/rs.3.rs-270635/v1.

40. Visscher AD, Sutton B, Sutton T. Second-wave dynamics of COVID-19: Impact of behavioral changes, immunity loss, new strains, and vaccination. Res Square. https://doi.org/10.21203/rs.3.rs-195879/v1.

41. Fujimoto $A B$, Yildirim I, Keskinocak P. Significance of SARS-CoV-2 Specific Antibody Testing during COVID-19 Vaccine Allocation. medRxiv. https://doi. org/10.1101/2021.01.28.21250721.

42. Shayak B, Sharma MM. COVID-19 Spreading Dynamics with Vaccination Allocation Strategy, Return to Normalcy and Vaccine Hesitancy. medRxiv. https://doi.org/10.1101/2020.12.10.20247049.

43. Bruningk SC, Klatt J, Stange M, Mari A, Brunner M, Roloff TC. et al, Determinants of SARS-CoV-2 transmission to guide vaccination strategy in a city. medRxiv. https://doi.org/10.1101/2020.12.15.20248130. 
44. Ayoub HH, Chemaitell H, Makhoul M, Al Kanaani Z, Al Kuwari E, Butt AA, et al. Epidemiological impact of prioritizing SARS-CoV-2 vaccination by antibody status: Mathematical modeling analyses. medRxiv. https://doi.org/1 0.1101/2021.01.10.21249382.

45. Castonguay FM, Blackwood JC, Howerton E, Shea K, Sims C. Sanchirico JN, Spatial Allocation of Scarce Vaccine and Antivirals for COVID-19. medRxiv. https://doi.org/10.1101/2020.12.18.20248439.

46. Fuady A, Nuraini N, Sukander KK, Lestari BW. Targeted Vaccine Allocation Could Increase the COVID-19 Vaccine Benefits Amidst Its Lack of Availability: A Mathematical Modeling Study in Indonesia. Vaccines. 2021;9(5):462.

47. Guerstein S, Romeo-Aznar V, Dekel M, Miron O, Davidovitch N, Puzis R, et al. Optimal strategies for combining vaccine prioritization and social distancing to reduce hospitalizations and mitigate COVID19 progression. medRxiv. https://doi.org/10.1101/2020.12.22.20248622.

48. Kirwin E, Rafferty E, Harback K, Round J, McCabe C. A Net Benefit Approach for the Optimal Allocation of a COVID-19 Vaccine. medRxiv. https://doi.org/1 0.1101/2020.11.30.20240986.

49. Wouters OJ, Shadlen KC, Salcher-Konrad M, Pollard AJ, Larson HJ, Teerawattananon Y, et al. Challenges in ensuring global access to COVID-19 vaccines: production, affordability, allocation, and deployment. Lancet. 2021; 397(10278):1023-934.

50. World Health Organization. WHO guide for standardization of economic evaluations of immunization programmes. https://apps.who.int/iris/bitstrea m/handle/10665/329389/WHO-IVB-19.10-eng.pdf. Accessed 8 June 2021.

\section{Publisher's Note}

Springer Nature remains neutral with regard to jurisdictional claims in published maps and institutional affiliations.

\section{Ready to submit your research? Choose BMC and benefit from:}

- fast, convenient online submission

- thorough peer review by experienced researchers in your field

- rapid publication on acceptance

- support for research data, including large and complex data types

- gold Open Access which fosters wider collaboration and increased citations

- maximum visibility for your research: over $100 \mathrm{M}$ website views per year

At $\mathrm{BMC}$, research is always in progress.

Learn more biomedcentral.com/submissions 